\title{
MER Use and Prolonged Surgical Duration May Not Be Risk Factors for Pneumocephalus Formation During DBS
}

Guglielmo less ( $\sim$ guglielmoiess@gmail.com )

University of Milan

Giulio Bonomo

Istituto Neurologico Carlo Besta

Vincenzo Levi

Istituto Neurologico Carlo Besta

Domenico Aquino

Istituto Neurologico Carlo Besta

Edvin Zekaj

Istituto Ortopedico Galeazzi

Federica Mezza

University of California, Los Angeles

Domenico Servello

Istituto Ortopedico Galeazzi

\section{Research Article}

Keywords: Deep Brain Stimulation, pneumocephalus, brain shift, surgical duration, time, MER

Posted Date: February 3rd, 2022

DOI: https://doi.org/10.21203/rs.3.rs-1300749/v1

License: (c) (i) This work is licensed under a Creative Commons Attribution 4.0 International License.

Read Full License 


\section{Abstract}

Purpose: Although only recently directional leads have proven their potential to compensate for suboptimally placed electrodes, optimal lead positioning remains the most critical factor in degerming Deep Brain Stimulation (DBS) outcome. Pneumocephalus is a recognized source of error, but the factors that contribute to its formation are still a matter of debate. Among these, operative time is one of the most controversial. Because cases of DBS performed with Microelectrode Recordings (MER) are affected by a significant increase in surgical length, it is useful to test for a difference in volumes of air in groups of patients who underwent MER during DBS and those who did not.

Methods: 73 and 21 patients who underwent DBS from two different Institutes were analyzed for the presence of postoperative pneumocephalus. The volumes of subdural air of patients who underwent the procedure with the use of microelectrode recordings were compared with those who underwent simple image-guided placement using the Mann-Whitney-U test. A correlation was also tested between surgical operative length and volume of air in a subset of patients.

Results: The volumes of air of cases in which MER were utilized were not statistically different from those performed without $(p=0.292)$. In a similar manner, surgical duration did not predict air volume. Side of surgery (monolateral vs. bilateral) also failed to demonstrate a statistically significant difference $(p=$ 0.075), as well as age.

Conclusion: operative time is probably not a significant parameter influencing pneumocephalus' formation.

\section{Introduction}

Deep brain stimulation (DBS) has become an established surgical procedure for the symptomatic treatment of several neurologic and psychiatric disorders such as Parkinson's disease (PD), essential tremor, dystonia, obsessive-compulsive disorder and Tourette's syndrome [1, 2, 3].

DBS success greatly depends on different factors, the most important of which being accurate lead placement $[4,5,6]$. Although the rapid advances in neuroengineering have increased the precision of the procedure, considerable discrepancy across patient's cohorts still exists. Two meta-analyses suggest a $45 \%$ rate of lead misplacement with consequent suboptimal therapeutic response $[7,8]$.

The most well-known and debated problem concerning electrode displacement in DBS is represented by the potential intraoperative brain shift caused by CSF leakage following burr hole creation and by consequent air entry in the skull [9]. This phenomenon is called pneumocephalus and, in turn, is thought to provoke unwanted transposition of brain structures.

Some factors such as patient age, cerebral atrophy and use of fibrin glue to cover burr holes may influence CSF loss and brain shift have been examined by different investigators [10,11]. Nevertheless, 
not much is known whether and to which extent operative time has to be considered a significant factor causing air entry during DBS. To date, very few studies have analyzed such correlation, and importantly, those have reported conflicting results $[10,12]$.

Because time is, to some extent, a surgeon-controllable aspect of DBS procedures, if a positive correlation is to be found, this could have an important impact on intraoperative decision-making: use of MER (microelectrode recordings) for example, the usefulness of which is still a matter of debate, significantly prolongs mean operative time. Although only recently, novel directional lead designs have proven their potential to compensate (at least partially) for sub-optimal electrode positioning, proper lead placement remains the mainstay of this treatment.

We present two cohorts from two different Institutes, composed of 73 and 21 individuals respectively who underwent DBS procedures under stereotactic conditions. Our primary objective was to investigate whether prolonged surgical duration poses the patient at risk of developing pneumocephalus during DBS.

\section{Material And Methods}

This is an observational retrospective study which includes a total of 94 DBS procedures, 73 of which performed at IRCCS Istituto Neurologico Carlo Besta (Institute 1) from 2014 to 2019 and the remaining 21 at IRCCS Istituto Ortopedico Galeazzi (Institute 2) from 2020 to 2021. Data was extrapolated from operative reports and medical records and comprised information such as surgical length of time, patient age, gender and diagnosis, DBS target, side (monolateral or bilateral), and use of intraoperative electrophysiological recordings (which included both microelectrode recordings and macrostimulation).

Because the surgical techniques (which are described briefly below) used in the two institutions were different, we decided to perform two separate analyses.

Pneumocephalus volumes were estimated based on an early postoperative CT scan (which was part of the routine practice) at IRCCS Istituto Neurologico Carlo Besta, while were calculated by two intraoperative CT scans at IRCCS Istituto Ortopedico Galeazzi carried out immediately after placing the first, and second electrode respectively (i.e., prior securing the definitive leads).

Similarly, it is important to note that operative lengths reported in the surgical reports of the two institutions differed for what concerns the starting time: while Institution 1 included also time between anesthetic induction and beginning of operation, Institution 2 reported solely operative length from the moment of the first skin incision to the final skin suture.

Surgeries were performed for different neurological and psychiatric conditions; more specifically: Parkinson's disease, dystonia, essential tremor, obsessive-compulsive disorder, major depression and cluster headache. Informed consent was obtained from all individual participants included in the study. The local ethical committees (IRCCS Istituto Neurologico Carlo Besta, Milan, Lombardia, Italy and IRCCS Istituto Ortopedico Galeazzi, Milan, Lombardia, Italy) approved the study. 
Volumes of pneumocephalus (figure 1) were calculated using the following steps: i) conversion of DICOM CT files in NiFTI format with dcm2nii (https://people.cas.sc.edu/rorden/mricron/dcm2nii.html); ii) threshold of the CT NiFTI files in order to create a binary brain and skull mask. The threshold was selected for each single case. This and the following steps were implemented in Matlab2017a (www.mathworks.com); iii) inversion (i.e. 1-mask) of the created mask to set at 1 the regions to be selected; iv) selection of the volumes to be estimated with a GUI Matlab developed in our Institute. Briefly, the GUI allows to select a single voxel of the binary volume and, with morphological operators, it extracts it from the entire volume; v) Volume estimation was obtained as the number of the extracted voxels multiplied for the volume of a single one of them.

\section{Statistical analysis}

Descriptive statistics (mean and $95 \% \mathrm{Cl}$ standard deviation) were used for continuous variables and frequency for categorical. We tested continuous variables for normality with the Shapiro-Wilk test and obtained statistically significant values for the distributions of volume and age of Istituto Neurologico Carlo Besta's patients, while all other variables did not reach significance and were therefore considered as normally distributed. Moreover, because Institute 1 volume's distribution had a considerable positive skewness $(1.279 \pm 0.28)$, median was preferred to mean as a measure of central tendency.

The Mann Whitney U-test was adopted to compare volumes for different groups of patients (monolateral vs bilateral, use of recordings vs direct image-based targeting). Variables that followed a normal distribution were compared using independent samples t-test. Correlation analysis was performed with Pearson's $r$ and Spearman's $\rho$ to detect any significant association between volume of air and surgical time and between volume and patient age. Chi-squared tests with $2 \times 2$ contingency tables were adopted to compare use of intraoperative recordings between the two groups formed by individuals who had either monolateral or bilateral implants.

Multiple linear regression analysis was employed to predict the volume of air of Institute 2. The predictor variables used were time, age and side (monolateral vs bilateral). Multiple linear regression was chosen to evaluate the effect of two continuous predictors and one dichotomous predictor simultaneously. The full model was confronted with an intercept model only by means of chi-square test. Since we were concerned about possible multicollinearity (which could have possibly hampered the analysis) between the independent variables, we performed multicollinearity diagnostics and obtained Variance Inflation Factor (VIF) scores lower than 2 (which were deemed acceptable).

All p-values reported are two-tailed and a $p<0.05$ was considered statistically significant.

Calculations and histograms were made using SPSS (IBM Corp. 2020 Release, IBM SPSS Statistics for MacOs, Version 26.0).

\section{Surgical technique at IRCCS Istituto Neurologico Carlo Besta}


Surgical procedure is thoroughly described elsewhere [13]. Depending on the target and on the degree of patient collaboration, DBS procedure is performed either with the patient awake or under general anesthesia. The day of the surgery a stereotactic computerized tomography (CT) scan is performed, and its images are merged with those of a preoperative magnetic resonance (MR). Final coordinates are calculated on the neuronavigation system (Stealth Station Treon Sofamor Danek, Medtronic Inc. Minneapolis, MN, USA) by adapting the individual patient anatomy to a probabilistic stereotactic digitalized atlas. A small burr hole of $5 \mathrm{~mm}$ diameter is drilled, and a rigid cannula is subsequently introduced after opening the dura mater. The cannula is initially placed $15 \mathrm{~mm}$ above the estimated target. At this point of the procedure, to limit cerebrospinal fluid leaks, a fibrin sealant is employed in the burr hole.

Microelectrode recordings (MER) are obtained using a high impedance microelectrode with 0.5-mm steps until $1 \mathrm{~mm}$ beyond the target along the single desired trajectory. Based on microrecordings localizing criteria, the definitive electrode (Medtronic Inc. Minneapolis, MN, USA; St. Jude Inc., St. Paul MN, USA) is then positioned at target using the same rigid cannula, after withdrawing the microelectrode.

Bipolar macrostimulation is next briefly performed to test clinical response in the awake patient.

Depending on whether MER and macrostimulation show optimal placement, the final electrode is secured using biological glue and with a titanium microplate.

If MER and/or clinical testing suggest an inaccurate electrode placement, a second trajectory is used (using the same burr hole) and the procedure is repeated again. A single trajectory was required in $70 \%$ of our DBS procedures, two trajectories in $20 \%$ and three or more in $10 \%$ of surgeries.

\section{Surgical procedure used at IRCCS Istituto Ortopedico Galeazzi}

On the day before surgery, a brain MRI is performed which consists in a volumetric gadolinium enhanced T1 sequence and axial T2 or DPI images. On the day of surgery after positioning the CRW stereotactic frame, the patient undergoes a stereotactic CT scan. MRI and stereotactic CT scan Images are transferred to the Brainlab Neuronavigation System and subsequently fused together. At this point a direct targeting strategy based on MRI images is performed. The patient is therefore positioned in the operating table integrated with the AIRO system with the head fixed on the CRW frame components. The operating table is shifted inside the AIRO, by applying table movements in order to set up the patient in the most comfortable position. Thereafter, a transparent drape is positioned and anchored with medical adhesive tapes to the AIRO. The frame ring is fixed, and the surgical procedure begins.

A linear skin incision and a 14-mm burr hole created with a high-speed drill are performed centered on the desired trajectory. A 4-mm diamond high-speed drill is utilized to drill out the external cranial bone and to fashion an optimal allocation for the burr hole cap. 
Absorbable hemostat, bone dust and fibrin glue are used to seal the burr hole after introducing the rigid cannula. Three microelectrodes are used for MER and advanced $0.5 \mathrm{~mm}$ every 30 seconds starting 10 $\mathrm{mm}$ above the target and penetrating 1-3 $\mathrm{mm}$ below. After completing micro recordings, macro stimulation is performed in order to observe for any eventual adverse events or signs of clinical improvement. After choosing the optimal target, the lead is positioned and fixed with a burr hole cap. Once electrodes are positioned, an intraoperative CT scan is obtained with the aid of AIRO with the patient in a horizontal position and a gait entry of $0^{\circ}$. Lasers of the AIRO are used to evaluate the craniocaudal and medio-lateral extension of the $\mathrm{CT}$ and the first and final acquiring positions are registered. At this point a scout image is obtained to confirm the correct extension of intraoperative CT scan images. If the scout image is in a suboptimal position, the initial and final positions are acquired again until the scout includes the area of interest. At this point the intraoperative CT scan is completed. The images are transferred to the Neuronavigation Brainlab software where they are fused with pre-operative images. If an error higher than $2 \mathrm{~mm}$ is noted, the leads are repositioned.

\section{Results}

Institute 1 cohort's consisted of 32 females and 41 males (Table 1). Mean patient's age was 48.29 years (SD \pm 16.93 , range 8-73).

Cohort's mean and median of intracranial air volume were 19.70 (SD \pm 26.12$)$ and 3.26 (range 0-93.03) $\mathrm{cm} 3$ respectively. Patient's mean age was 48.29 years ( $\mathrm{SD} \pm 16.93$, range 8-73). In 53 (72.6\%) procedures intraoperative recordings were utilized, while in the remaining 20 (27.4\%) were not. 55 subjects $(75.3 \%)$ had bilateral electrodes implantations, while $18(24.7 \%)$ had monolateral ones. Although bilateral implants had a higher median volume (9.28 vs $1.32 \mathrm{~cm} 3$ ), no significant difference was found when comparing monolateral implants with bilateral $(p=0.075)$.

Mean operative time was 130.70 minutes (SD \pm 51.83 ) and, as expected, was significantly different between patients who underwent recordings and those who did not, utilizing independent-samples t-Test $(p<0.0001)$.

Although subjects undergoing recordings presented a slightly higher volume of intracranial air on postoperative CT than those operated without, such comparison did not reach statistical significance $(\mathrm{p}=$ 0.292).

Moreover, it is important to note that in bilateral DBS 78\% (46/59) patients had intraoperative MER while only $38 \%(7 / 18)$ with monolateral surgeries were tested with recordings $(p<0.0001)$.

Furthermore, operative time and air volume had a correlation of 1.60 , a value not yet sufficient to indicate statistical significance $(p=0.167)$. Similarly, age did non correlate with pneumocephalus $(\rho=0.104, p=$ $0.381)$. 
Table 1

Table with descriptive statistics of the case series of Institute 1. STN, Subthalamic Nucleus; GPI, Globus

Pallidus Internus; VIM, Ventral Intermediate Nucleus; HPT, Hypothalamus; BNST, Ben Nucleus Stria Terminalis; SCG24, subgenual cingulate gyrus 24; SD, Standard Deviation.

\begin{tabular}{|c|c|c|}
\hline Parameter & Use of MER & Without use of MER \\
\hline $\begin{array}{l}\text { Gender, } n[F=\text { females; } \\
\text { M=males] }\end{array}$ & $23 \mathrm{~F} ; 30 \mathrm{M}$ & $8 \mathrm{~F} ; 12 \mathrm{M}$ \\
\hline Age, years [mean \pm SD] & $46.70 \pm 16.84$ & $52.50 \pm 16.87$ \\
\hline Side, $\mathrm{n}[\%]$ & $\begin{array}{l}46 \text { Bilateral [86.8\%]; } 7 \text { Monolateral } \\
\text { [13.2\%] }\end{array}$ & $\begin{array}{l}9 \text { Bilateral [45\%]; } 11 \text { Monolateral } \\
{[55 \%]}\end{array}$ \\
\hline Target, $\mathbf{n}$ & $\begin{array}{l}30 \text { STN; } 17 \text { GPI; } 3 \text { VIM; } 2 \text { HPT; } 1 \\
\text { SCG24 }\end{array}$ & $\begin{array}{l}2 \text { STN; } 1 \text { GPI; } 13 \text { VIM; } 3 \text { BNST; } 1 \\
\text { SCG24 }\end{array}$ \\
\hline $\begin{array}{l}\text { Volume, cm3 [median; } \\
\text { range] }\end{array}$ & $3.26 ; 0-93.03$ & $1.43 ; 0-53.11$ \\
\hline Time, min $[$ mean \pm SD] & $148.89 \pm 41.43$ & $82.50 \pm 45.99$ \\
\hline
\end{tabular}

Institute 2 cohort's included 21 patients (descriptive statistics are summarized in Table 2). All surgeries were performed with the use of intraoperative recordings. No association between volume and time was found using Pearson's correlation $(\rho=0.119, p=0.616)$. With multiple linear regression analysis none of the independent variables appeared to be a significant predictor (Table 3).

Table 2

Table with descriptive statistics of the case series of Institute 2. Differently from the other center, all cases underwent MER recordings. STN, Subthalamic Nucleus; GPI, Globus Pallidus Internus; SD, Standard Deviation.

\begin{tabular}{|ll|}
\hline Parameter & \\
\hline Gender, $\mathrm{n}$ [F=females; $\mathrm{M}=$ males] & $10 \mathrm{~F} ; 11 \mathrm{M}$ \\
\hline Age, years [mean $\pm \mathrm{SD}]$ & $59.75 \pm 10.31$ \\
\hline Side, $\mathrm{n}[\%]$ & 19 Bilateral; 2 Monolateral \\
\hline Target, $\mathrm{n}$ & $13 \mathrm{STN} ; 8 \mathrm{GPI}$ \\
\hline Volume, cm3 [median; range] & $26.35 ; 0-76.10$ \\
\hline Time, min [mean \pm SD] & $159.71 \pm 26.46$ \\
\hline
\end{tabular}


Table 3

Multiple linear regression analyzing the simultaneous effects of Age (in years), Time (in minutes) and Side (monolateral vs. bilateral) on the total volume of air (in $\mathrm{cm} 3$ ) of the patients operated in Institute 2.

\begin{tabular}{|lll|}
\hline Parameter & Unstandardized B & $\mathbf{P}$ \\
\hline Time & -0.09 & 0.644 \\
\hline Age & 0.452 & 0.263 \\
\hline Side & -8.77 & 0.681 \\
\hline
\end{tabular}

\section{Discussion}

Brain shift is a well-known phenomenon, and it is now clear that the amount of subdural air entered in the skull during the procedure has a negative impact on the precision of both navigation and stereotactic systems based on preoperative image data $[14,15,16]$. Long-term efficacy of DBS may rely on even a modest amount of pneumocephalus $[11,12,15,17,18]$. Brain shift tends to displace targets in the posterior and superior directions, resulting in aberrant pathway activation from pre-operative predicted plan [17]. Hill et al. calculated a median brain surface shift after incising the dura ranging from 0.3 to 7.4 $\mathrm{mm}$. Other investigators reported a deviation of up to $4 \mathrm{~mm}$ shift of subcortical structures $[10,18,19]$.

It is a common belief among surgeons that air influx, rather than being a fast process, takes place over time. As a consequence, longer operative lengths are thought to come at a cost of greater amounts of pneumocephalus. To counteract further air accumulation over time, many centers adopted different strategies like the use of burr hole sealants (like fibrin glue or bone wax), reducing burr hole diameter, and direct dural puncture to reduce CSF egress [20, 21, 22]. Yet, although some studies reported a decrease in air inflow, such approaches did not prevent pneumocephalus from forming $[14,22]$. This can be explained by the fact that most of the cerebrospinal fluid loss (which is subsequently replaced by subdural gas) is likely to occur immediately after incising the dura. Indeed, considering fluid dynamics, after burr hole trepanation and dural piercing, the volume of CSF above this point will flow out, pushed by gravity.

Considering that most DBS are performed with the patient lying flat in a supine position (or with slight back upward inclination), the amount of fluid that is lost during surgery according to this theory coincides with that filling subdural spaces from the most posterior point of the burr holes to the frontal poles.

In line with this concept, we found no evidence of association between operation time and volume of pneumocephalus at Institute 1, when using a bivariate correlation. Similar findings have been reported by Ko et al [23]. However, these results have the important flaw of considering time from anesthetic records which, by definition, does not automatically mirror solely the amount of time the dura is open.

To address this problem, we adopted two different approaches. By means of the first approach, we compared patients of Institute 1 who underwent DBS procedures that included intraoperative recordings 
with those who were implanted by direct image-based targeting. Because the use of recordings inevitably prolongs the operative time while the dura is opened (which was confirmed by the significant difference in mean operative time between the two groups), such strategy is robust to this bias. However, the group of patients who underwent recordings had only a modestly higher amount of intracranial air, which was not statistically significant when performing the confrontation between the two groups.

Further complicating the matter, it has to be pointed out that a significantly higher proportion of patients that were recorded during the procedure had bilateral implantations compared to the group of individuals who had direct image-implantation. Considering that bilateral implantations presented a greater mean air volume than monolateral, the side of surgery could potentially act as a confounding factor. From a theoretical point of view, it is possible that the falx could act as a physical barrier preventing part of liquoral egress from the side controlateral to the burr hole to the outside of the skull during monolateral implants. Nonetheless, because right and left subarachnoid spaces are in direct communication, such block has limited influence on the total amount of CSF lost with the patient in supine position. In support of this thesis, the difference in volume of gas between monolateral and bilateral implants did not reach statistical significance in our study.

With the second approach, we performed a separate analysis on patients of Institute 2 , where the operative times in the surgical reports are reported from the moment of the first skin incision to the termination of the skin suture. Because the amount of time needed to perforate the dura from the initial incision may be considered (in a first approximation) similar, such computation is more robust to this bias. Indeed, also with this second approach no correlation was found between volume and time, confirming previous results. It is important to note however, that the number of cases was significantly lower compared to Institute 1.

Another important problem is the one related to brain atrophy. As mentioned earlier, because the volume of CSF above the burr holes is the one being subject to outflow, patients with higher degrees of brain atrophy are at increased risk for developing pneumocephalus, as they have a higher proportion of $\mathrm{CSF} /$ brain parenchyma than their normotrophic counterparts [24].

Because CSF egress is a gravity-dependent phenomenon, planning the surgical procedure placing the burr holes at the highest point of the skull, may be a useful solution to minimize the quantity of gas which fills the subdural space [9]. Although the position of the burr hole can vary only up to a certain point, it is possible to regulate the inclination of the head by changing the patient positioning. Utilizing a semisitting position may be advantageous from this point of view because it relocates the burr holes on top of the skull. In addition to minimizing the quantity of CSF on top of the burr hole, the semi-sitting position changes the orientation of the brain in relation to the force of gravity. Consequently, air would accumulate on top of the skull concavity resulting in a superior-to-inferior force.

\section{Conclusion}


In our study, operative time and the use of intraoperative microelectrode recordings do not seem to significantly influence the formation of pneumocephalus during deep brain stimulation procedures. Minimizing the amount of air entry in the skull during DBS procedures is crucial to guarantee accurate lead placement and creating burr holes at the highest points of the skull with respect to patient intraoperative positioning may represent an effective way to achieve this goal.

\section{Declarations}

Funding: This research received no specific grant from any funding agency in the public, commercial, or not-for-profit sectors.

Conflicts of interest: The authors have no conflicts of interest to declare that are relevant to the content of this article.

Data availability: Data is available from the Corresponding Author (GI) upon reasonable request.

Code availability: Not applicable

Ethical approval: All procedures performed in studies involving human participants were in accordance with the ethical standards of the institutional and/or national research committee and with the 1964 Helsinki declaration and its later amendments or comparable ethical standards

Informed consent: Informed consent was obtained from all individual participants included in the study.

Consent for publication: The authors affirm that human research participant provided informed consent for publication of the image in Figure 1.

Author contribution: Material preparation and data collection were carried out by Guglielmo less and Giulio Bonomo.

Conceptualization and design were performed by Guglielmo less, Domenico Aquino and Vincenzo Levi, while the first draft of the manuscript was written by Guglielmo less and all authors commented on previous versions of the manuscript. Guglielmo less also carried out statistical analysis. Domenico Servello reviewed and validated the final manuscript.

\section{References}

1. Sugiyama, K., et al. The present indication and future of deep brain stimulation. Neurologia medicochirurgica, 55(5), 416-421 (2015).

2. Servello, D., Zekaj, E., Saleh, C., Lange, N., \& Porta, M. Deep brain stimulation in Gilles de la Tourette syndrome: what does the future hold? A cohort of 48 patients. Neurosurgery, 78(1), 91-100 (2016).

3. Fisher, R. S., \& Velasco, A. L. Electrical brain stimulation for epilepsy. Nature Reviews Neurology, 10(5), 261-270 (2014). 
4. Volkmann, J., Moro, E., \& Pahwa, R. Basic algorithms for the programming of deep brain stimulation in Parkinson's disease. Movement disorders: official journal of the Movement Disorder Society, 21(S14), S284-S289 (2006).

5. Walter, B. L., \& Vitek, J. L. Surgical treatment for Parkinson's disease. The Lancet Neurology, 3(12), 719-728 (2004).

6. Machado, A., et al. Deep brain stimulation for Parkinson's disease: surgical technique and perioperative management. Movement disorders: official journal of the Movement Disorder Society, 21(S14), S247-S258 (2006).

7. Okun, M. S., et al. Management of Referred Deep Brain Stimulation Failures: A Retrospective Analysis From Two Movement Disorder Centers: 818. Neurosurgery, 57(2), 401 (2005).

8. Rolston, J. D., Englot, D. J., Starr, P. A., \& Larson, P. S. An unexpectedly high rate of revisions and removals in deep brain stimulation surgery: analysis of multiple databases. Parkinsonism \& related disorders, 33, 72-77 (2016).

9. Toung, T. J., et al. Pneumocephalus: effects of patient position on the incidence and location of aerocele after posterior fossa and upper cervical cord surgery. Anesthesia and analgesia, 65(1), 6570 (1986).

10. Halpern, C. H., Danish, S. F., Baltuch, G. H., \& Jaggi, J. L. Brain shift during deep brain stimulation surgery for Parkinson's disease. Stereotactic and functional neurosurgery, 86(1), 37-43 (2008).

11. Miyagi, Y., Shima, F., \& Sasaki, T. Brain shift: an error factor during implantation of deep brain stimulation electrodes. Journal of neurosurgery, 107(5), 989-997 (2007).

12. Elias, W. J., Fu, K. M., \& Frysinger, R. C. Cortical and subcortical brain shift during stereotactic procedures. Journal of neurosurgery, 107(5), 983-988 (2007).

13. Franzini, A., Messina, G., Cordella, R., Marras, C., \& Broggi, G. Deep brain stimulation of the posteromedial hypothalamus: indications, long-term results, and neurophysiological considerations. Neurosurgical focus, 29(2), E13 (2010).

14. Coenen, V. A., Abdel-Rahman, A., McMaster, J., Bogod, N., \& Honey, C. R. Minimizing brain shift during functional neurosurgical procedures-a simple burr hole technique that can decrease CSF loss and intracranial air. Central European Neurosurgery-Zentralblatt für Neurochirurgie, 72(04), 181-185 (2011).

15. Van den Munckhof, P., et al. Postoperative curving and upward displacement of deep brain stimulation electrodes caused by brain shift. Neurosurgery, 67(1), 49-54 (2010).

16. Sillay, K. A., et al. Perioperative brain shift and deep brain stimulating electrode deformation analysis: implications for rigid and non-rigid devices. Annals of biomedical engineering, 41(2), 293-304 (2013).

17. Choi, K. S., et al. Impact of brain shift on subcallosal cingulate deep brain stimulation. Brain stimulation, 11(2), 445-453 (2018).

18. Khan, M. F., Mewes, K., Gross, R. E., \& Škrinjar, O. Assessment of brain shift related to deep brain stimulation surgery. Stereotactic and functional neurosurgery, 86(1), 44-53 (2008). 
19. Obuchi, T., et al. Direction and predictive factors for the shift of brain structure during deep brain stimulation electrode implantation for advanced Parkinson's disease. Neuromodulation: Technology at the Neural Interface, 11(4), 302-310 (2008)

20. Sharim, J., Pezeshkian, P., DeSalles, A., \& Pouratian, N. Effect of cranial window diameter during deep brain stimulation surgery on volume of pneumocephalus. Neuromodulation: Technology at the Neural Interface, 18(7), 574-579 (2015).

21. Piacentino, M., et al. Pneumocephalus in subthalamic deep brain stimulation for Parkinson's disease: a comparison of two different surgical techniques considering factors conditioning brain shift and target precision. Acta Neurochirurgica, 163(1), 169-175 (2021).

22. Beggio, G., et al. Techniques for pneumocephalus and brain shift reduction in DBS surgery: a review of the literature. Neurosurgical review, 43(1), 95-99 (2020).

23. Ko, A. L., Magown, P., Ozpinar, A., Hamzaoglu, V., \& Burchiel, K. J. Asleep deep brain stimulation reduces incidence of intracranial air during electrode implantation. Stereotactic and functional neurosurgery, 96, 83-90 (2018).

24. Azmi, H., Machado, A., Deogaonkar, M., \& Rezai, A. Intracranial air correlates with preoperative cerebral atrophy and stereotactic error during bilateral STN DBS surgery for Parkinson's disease. Stereotactic and functional neurosurgery, 89(4), 246-252 (2011).

\section{Figures}




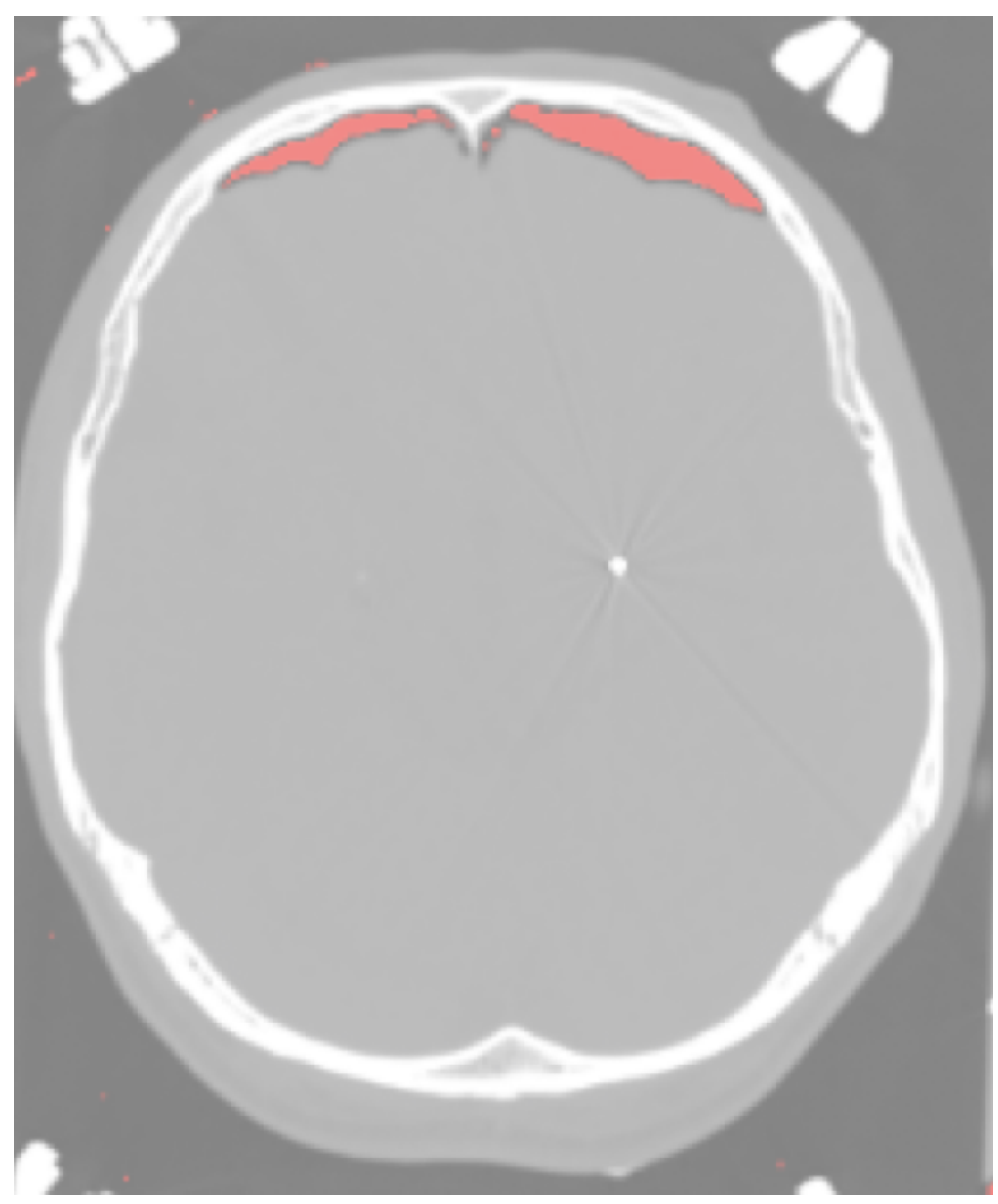

Figure 1

Image illustrating the volume of pneumocephalus calculated with the GUY Matlab developed in our Institute on the postoperative axial-CT images. The area drawn red in the frontal poles represents the intracranial air. 